\title{
Avascular necrosis of the head of the femur in a case of retroperitoneal fibrosis
}

\author{
B. A. KAMDAR \\ M.B., B.S., F.R.C.S. \\ Orthopaedic Unit, Department of Surgery, \\ Hammersmith Hospital and Royal Postgraduate Medical School, London W.12
}

\begin{abstract}
Summary
A case of avascular necrosis of the head of the femur is reported. The patient is known to suffer from retroperitoneal fibrosis. The possibility of an association between these two conditions is discussed.
\end{abstract}

IN a review of the medical literature since 1948 when retroperitoneal fibrosis was first described by Ormond, no case of avascular necrosis of the head of femur has been reported in association with it. A patient is reported here with retroperitoneal fibrosis who 10 years later presented with avascular necrosis of the head of the femur.

\section{Case report}

The patient is a woman aged 67 years, known to suffer from retroperitoneal fibrosis, first diagnosed in 1960. She had had a left nephrectomy for hydronephrosis and pyelonephritis of the left kidney in September 1959. She was well until December 1959. She then developed low backache and on 18 December 1959 developed oliguria. She was treated by emergency measures and a spontaneous diuresis followed; the serum electrolytes and urea were normal by 21 December. An excretion urogram on 1 January 1960 showed a right hydronephrosis; the ureter was narrowed at the level of L5 vertebra and a similar appearance had been noted on the left side in September 1959. The ESR was constantly raised. A provisional diagnosis of retroperitoneal fibrosis was made and a right nephropexy, ureterolysis and a retroperitoneal biopsy were performed on 2 January 1960 . She made satisfactory progress till November 1960 when oliguria developed. A right ureterostomy was performed as an emergency procedure which was later converted into an uretero-ileocystoplasty, at which time recurrence of retroperitoneal fibrosis was noted. Subsequently she developed oedema in both lower limbs which was controlled by regular use of diuretics.

Apart from occasional urinary tract infection the patient remained well until the end of July 1970, when she developed pain in her right groin and iliac fossa. She was admitted to the renal unit for assessment on 6 August 1970. The pain in her right groin settled with rest and as no urological cause for pain was found she was discharged home. However, the pain returned with increasing severity in October 1970 and the patient was referred to the orthopaedic clinic. At that time she could not bear any weight on her right hip; the movements of the right hip joint were extremely painful and grossly restricted. There was no history of previous trauma and no history of steroid therapy or the use of the methysergide group of drugs.

Radiographs of the right hip joint showed marked irregularity and depression of the supero-lateral part of the articular surface of the femoral head (Fig. 1). There was diminution of the joint space. Routine haematological investigations were normal. The ESR was $85 \mathrm{~mm} / \mathrm{hr}$. Though the appearances were consistent with avascular necrosis of the head of femur, infection could not be excluded. Aspiration of the hip was negative.

At operation the right femoral head and neck were excised and replaced by an Austin Moore's pros-

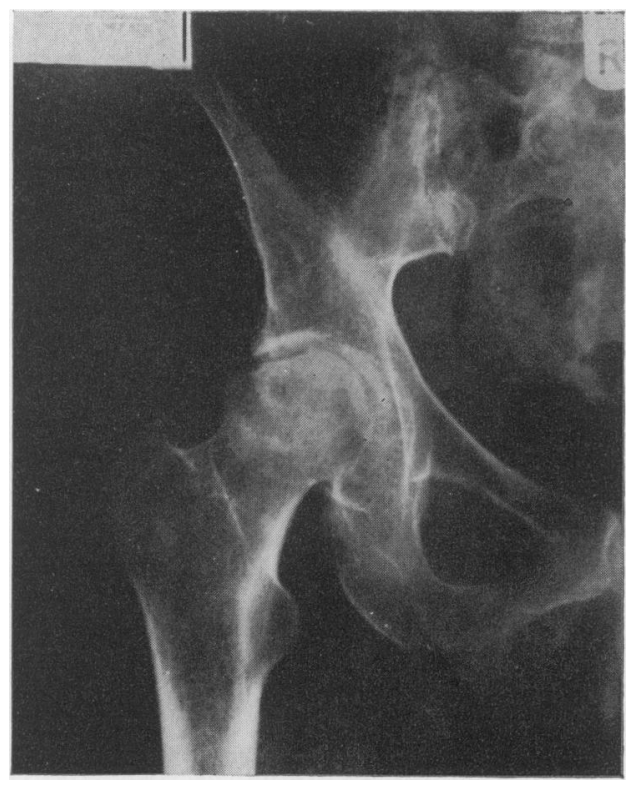

FIG. 1. Radiograph of the pelvis showing the avascular necrosis of the head of right femur 


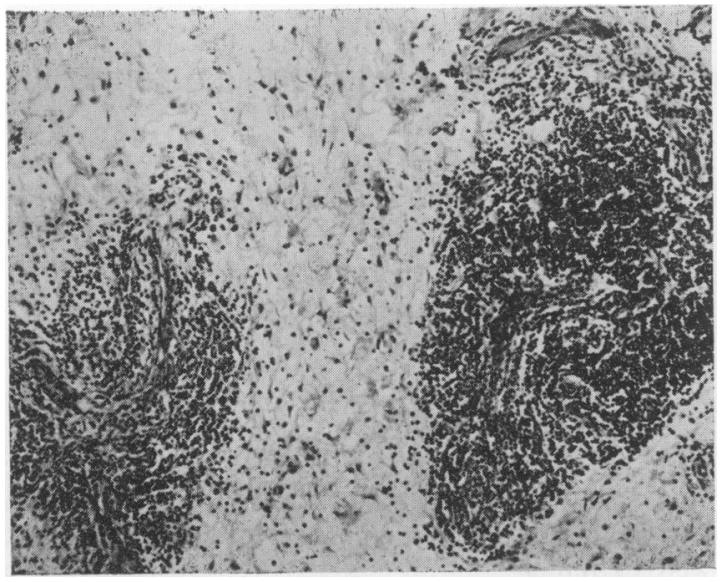

FIg. 2. Histology of the ligamentum teres and synovium, showing perivascular cuffing by lymphocytes, plasma cells and polymorphnuclear leucocytes.

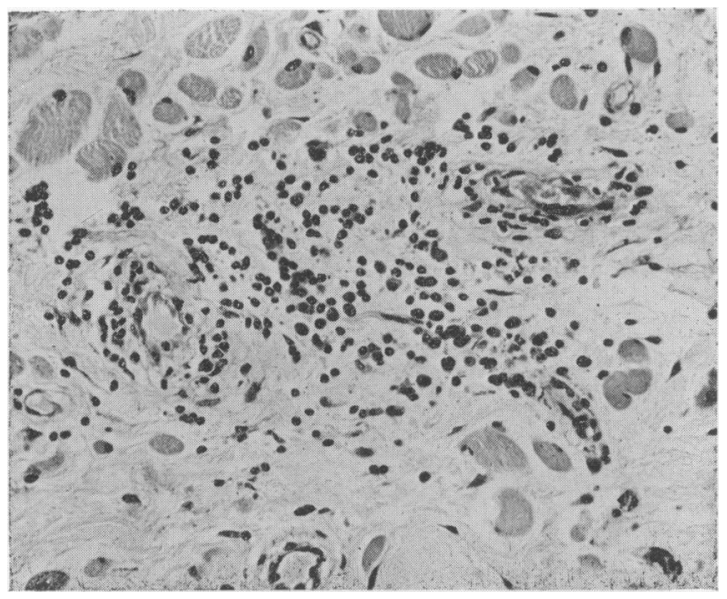

FIg. 3. Histology of the retroperitoneal tissue showing perivascular cuffing in some areas.

thesis. Convalescence of the patient was uneventful, and she was allowed to bear weight on the right lower limb 3 weeks after operation.

The head of the femur, synovial membrane, ligamentum teres and the capsule were examined. The articular surface of the head of femur was irregular, and beneath it there was an area of bone which was dense white in colour. This area of avascular bone (size $3.5 \times 5 \mathrm{~cm}$ ) was situated in the antero-lateral part of the head. Microscopic examination confirmed aseptic necrosis; the viable bone showed sclerosis and there was fibrosis of the medullary spaces.
Sections of the synovium and of the ligamentum teres (Fig. 2) showed a mild degree of chronic inflammatory cell infiltration and prominent cuffing by lymphocytes and plasma cells of many of the capillaries, venules and a few arterioles. There was endothelial swelling and infiltration by mononuclear cells and occasional polymorphnuclear neutrophils. There was no evidence of fibrinoid necrosis. The capsule showed no special features.

Histological examination of the retroperitoneal adipose and fibrous tissue (Fig. 3) showed a diffuse infiltration by lymphocytes, plasma cells, eosinophils and polymorphs. There was perivascular cuffing in some areas. In addition some new fibrous tissue had formed. The appearances were consistent with a diagnosis of retroperitoneal fibrosis.

\section{Discussion}

It cannot be concluded from one case that there is definite evidence of some association between retroperitoneal fibrosis and avascular necrosis of the head of femur, but the occurrence of the latter in a patient who has had retroperitoneal fibrosis for the past 11 years, and the presence of vasculitis in the tissues taken for biopsy from the retroperitoneum in 1960 and from the hip in 1970, does suggest a possible relationship between the two conditions. That the retroperitoneal disease in this $\omega$ case has been constantly active for the past 11 years is shown by the following facts: (1) continued in volvement of the right ureter by the progressive retroperitoneal fibrosis, leading to several operations between December 1959 and December 1960; (2) continually raised erythrocyte sedimentation rate for the past eleven years; (3) the development of oedema in both lower limbs, more pronounced on the left leg than the right leg. Venography demonstrated narrowing of the inferior vena cava at the level of the L4 vertebra.

\section{Acknowledgment}

I would like to thank Mr W. H. Stephenson for his encouragement in the preparation of this paper and for allowing me to publish the case which is under his care.

\section{Bibliography}

Chandler, F.A. (1948) Coronary disease of the hip. Journal of International College of Surgeons, 11, 34.

HAW, W. A. \& HAZARD, J.B. (1959) Sclerosing retroperi- o tonitis and sclerosing mediastinitis. American Journal of $\mathrm{N}$ Clinical Pathology, 32, 321.

KAY, R.G. (1963) Retroperitoneal vasculitis with perivascular fibrosis. British Journal of Urology, 35, 284.

Mitchisen, M.J. (1970) The pathology of idopathic retroperitoneal fibrosis. Journal of Clinical Pathology, 23, 681.

Merle D'Aubigne, R., Postel, M., Mazabrand, A., Messias, R. \& GUEGUEN, J. (1965) Idiopathic necrosis of femoral head in adults. Journal of Bone and Joint Surgery, $47 \mathrm{~b}, 612$. 
ORMOND, J.K. (1948) Bilateral ureteral obstruction due to envelopment and compression by an inflammatory retroperitoneal fibrosis. Journal of Urology, 19, 1072.

ORMOND, J.K. (1966) Idiopathic retroperitoneal fibrosis: an established clinical entity. Journal of American Medical Association, 174, 1661.

Patterson, R.J., Bickel, M.H. \& Dahlin, D.C. (1964) Idiopathic avascular necrosis of the head of femur. Journal of Bone and Joint Surgery, 46a, 267.
Phemister, D.B. (1934) Fractures of the neck of femur producing aseptic necrosis of the head of femur. Surgery, Gynaecology and Obstetrics, 159, 415.

Que, G.S. \& MAlldema, E. (1964). A case of retroperitoneal fibrosis presenting as a systemic collagen disease. American Journal of Medicine, 36, 220.

TRUETA, J. \& HARRISON, M.H.M. (1953) The normal vascular anatomy of the femoral head in ault men. Journal of Bone and Joint Surgery, 35b, 442.

\title{
An unexpected abdominal mass
}

\author{
MEIRION THOMAS* \\ M.B., B.S. \\ Masaka Hospital, Uganda
}

\begin{abstract}
Summary
An unusual complication of ectopic pregnancy, presenting during post-menopausal life, is reported. As hospital services become available to previously medically isolated communities, early diagnosis and treatment will make such cases as this rare.
\end{abstract}

\section{Case report}

A Baganda woman was admitted in February, 1971, complaining of a painless abdominal mass present for over 20 years. She had not menstruated for over 2 years but, like most women in rural Africa, was uncertain of her age. She was probably 50 years old. Of her three children, two had died in the first week of life, and only the third, a 30-year-old male, was alive and well. One year after the birth of her third child, she had had amenorrhoea for 6 months and she thought she was pregnant again. Uneventfully normal menstruation was resumed and she was subsequently sterile.

On abdominal examination, a very large mass, the size of a 20 week pregnancy, was palpated, arising centrally from the pelvis. On vaginal examination, the site of the mass was confirmed and was thought to be in continuity with the uterus. A preoperative diagnosis of calcified fibroids was made.

At laparotomy, the uterus was only slightly enlarged and studded with very small calcified fibroids. The right ovary was atrophic and there was a small right hydrosalpinx. Replacing the left ovary there was an oval, calcified mass, $20 \times 10 \mathrm{~cm}$, attached to the uterus by the left fallopian tube and

\footnotetext{
* Present address: Department of Surgery, Royal Postgraduate Medical School and Hammersmith Hospital, Ducane Road, London, W12 OHS.
}

left utero-ovarian ligament. The mass was densely adherent to small bowel and omentum. Adhesions were divided and a total hysterectomy and bilateral salpingo-oophorectomy was performed.

After operation, the mass was opened using a variety of orthopaedic instruments. The calcified capsule was 1-2 cm thick (Fig. 1). Some foetal parts were identified and removed (Fig. 2), but most of the skeleton had been incorporated into the capsule.

\section{Comment}

It was surprising to us that the patient denied any symptoms referable to a ruptured ectopic pregnancy.

The bone development suggested that the gestational age of the foetus was 5-6 months. Remarkably, this 'missed' ectopic had organized, and over a

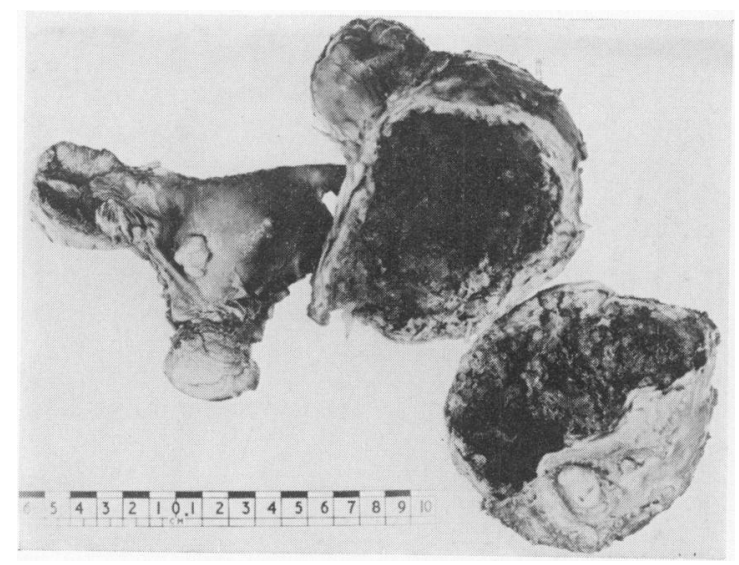

FIG. 1. Operative specimen described in the text. 